\title{
ESTUDO ESPECTROSCÓPICO EM ELUCIDAÇÃO ESTRUTURAL DE FLAVONOIDES DE Solanum jabrense AGRA \& NEE E S. paludosum MORIC.
}

\section{Tania Maria Sarmento da Silva*}

Departamento de Química, Universidade Federal Rural de Pernambuco, Rua Dom Manoel de Medeiros, s/n, 52171-900 Recife - PE, Brasil

\section{Mario Geraldo de Carvalho}

Departamento de Química, Instituto de Ciências Exatas, Universidade Federal Rural do Rio de Janeiro, BR 465, km 07, 23890-000 Seropédica - RJ, Brasil

\section{Raimundo Braz-Filho}

Setor de Química de Produtos Naturais, CCT, Universidade Estadual do Norte Fluminense, Av. Alberto Lamego, 2000, 28013-600 Campos dos Goytacazes - RJ, Brasil

\begin{abstract}
SPECTROSCOPY STUDY ON STRUCTURAL ELUCIDATION OF FLAVONOIDS FROM Solanum jabrense Agra \& Nee e $S$. paludosum Moric. The NMR (RMN $\left.{ }^{1} \mathrm{H},{ }^{13} \mathrm{C}, \mathrm{COSY}, \mathrm{HMQC}, \mathrm{HMBC}, \mathrm{NOE}-\mathrm{DIFF}, \mathrm{NOESY}\right)$ and mass spectra data analysis of sixteen flavonoids, including nine natural, 7- $O$-methylkanferol (ramnocitrin), 3,7-di- $O$-methylkanferol (kumatakenin), 3- $O$-methylquercetin, 3,7,3',4'-tetra- $O$-methylquercetin (retusin), 3,7,8,4'-tetra- $O$-methylgossipetin, 3,7,8,3',4'-penta- $O$-methylgossipetin, 7-O-methylapigenin (genkwanin), 3,7,8-tri- $O$-methylherbacetin, 7,4'- di- $O$-methylquercetin (ombuine), isolated from Solanum paludosum and $S$. jabrense, and seven prepared methyl and acetyl derivatives, are discussed according the substitution on the rings $\mathrm{A}, \mathrm{B}$ and $\mathrm{C}$.
\end{abstract}

Keywords: Solanum paludosum; Solanum jabrense; flavonoids.

\section{INTRODUÇÃO}

O estudo químico envolvendo as partes aéreas de Solanum paludosum Moric. e S. jabrense Agra \& Nee resultou no isolamento de 8 e 7 flavonoides, respectivamente. Em trabalhos anteriores relatouse a presença dos flavonoides 7-O-metilkanferol (ramnocitrina, 1), 3,7-di- $O$-metilkanferol (kumatakenina, 2), 3-O-metilquercetina (7), 3,7,3',4'-tetra- $O$-metilquercetina (retusin, 8), 3,7,8,4'-tetra- $O$ metilgossipetina (11), 3,7,8,3',4'-penta- $O$-metilgossipetina (12), 7-O-metilapigenina (genkwanina, 15), 3,7,8-tri- $O$-metilherbacetina (16) em $S$. paludosum ${ }^{1,2}$ e 1, 2, 7, 7,4'- di- $O$-metilquercetina (ombuína, 6), 8, 11 e 12 em $S$. jabrense. ${ }^{3}$ Destes 3,7,8,4'-tetra- $O$-metilgossipetina, 3,7,8,3',4'-penta- $O$-metilgossipetina, 7- $O$-metilapigenina, 7-O-metilkanferol e 7,4'- di-O-metilquercetina foram isolados pela primeira vez no gênero Solanum, apresentando, portanto, uma grande contribuição para o entendimento da quimiotaxonomia do gênero.

Muitos flavonoides ocorrem amplamente na natureza e é muito difícil obter em quantidade suficiente de fonte natural para identificação das estruturas. Os dados de RMN de muitos flavonoides hidroxilados, metoxilados e acetilados podem ajudar a identificar novos flavonoides isolados de fontes naturais baseados nos experimentos de RMN.

Uma vez que os flavonoides das espécis $S$. paludosum e $S$. jabrense foram isolados em quantidade razoável, este trabalho mostra a preparação dos derivados metilados 3 (7,4'-di- $O$-metilkanferol) e 9 (3, 5, 7, 3',4'-penta- $O$-metilquercetina), e acetilados 4 (3,7-di$O$-metil, 5,4'-di- $O$-acetilkanferol), 5 (5 7-di- $O$-metil, 3,5,4'-tri- $O$ acetilkanferol), 10 (3,7,3',4'-tetra- $O$-metil, 5- $O$-acetilquercetina), 13 (3,7,8,3',4'-penta- $O$-metil, 5- $O$-acetilgossipetina) e 14 (3,7,8,4'-tetra$O$-metil, 5,3'-di- $O$-metilgossipetina). Considerando que estes dados podem variar consideravelmente pela introdução de grupos hidroxila, metoxila e acetila nas estruturas dos flavonoides, faz-se uma discus-

*e-mail: sarmento@pesquisador.cnpq.br são dos dados espectroscópicos de RMN e massas obtidos de todos os flavonoides naturais e dos derivados preparados por reações de metilação e acetilação.

\section{PARTE EXPERIMENTAL}

\section{Procedimentos experimentais gerais}

Os espectros de $\mathrm{RMN}{ }^{1} \mathrm{H}$ e ${ }^{13} \mathrm{C}$ (1D e 2D) foram registrados em espectrômetros Bruker DRX-500 ( ${ }^{1} \mathrm{H}: 500 \mathrm{MHz} ;{ }^{13} \mathrm{C}$ : $\left.125 \mathrm{MHz}\right)$, Bruker AMX-300 ( $\left.{ }^{1} \mathrm{H}: 300 \mathrm{MHz} ;{ }^{13} \mathrm{C}: 75 \mathrm{MHz}\right)$, Bruker AC-200 $\left({ }^{1} \mathrm{H}\right.$ : $\left.200 \mathrm{MHz} ;{ }^{13} \mathrm{C}: 50 \mathrm{MHz}\right)$ e JEOL Eclipse-400 $\left({ }^{1} \mathrm{H}: 400 \mathrm{MHz} ;{ }^{13} \mathrm{C}: 100\right.$ $\mathrm{MHz}$ ) em tubos de $5 \mathrm{~mm}$, utilizando DMSO-d ${ }_{6}, \mathrm{CDCl}_{3}$ ou acetona$\mathrm{d}_{6}$ como solvente e TMS como referência interna. Utilizaram-se os programas de pulsos da Bruker $\left[\mathrm{zg} 30\left({ }^{1} \mathrm{H}\right)\right.$, zgpg30 $\left({ }^{13} \mathrm{C} \mathrm{BBHD}\right)$, dept135 $\left({ }^{13} \mathrm{C}\right.$ DEPT), cosygs $\left({ }^{1} \mathrm{H}-{ }^{1} \mathrm{H}\right.$ COSY), inv4gstp (HMQC), inv4gsllmd (HMBC) e noesygpph $\left({ }^{1} \mathrm{H}-{ }^{1} \mathrm{H}\right.$ NOESY)] com probe dual ${ }^{13} \mathrm{C} /{ }^{1} \mathrm{H}$ para detecção normal $\left({ }^{13} \mathrm{C}\right)$ ou com detecção inversa para $\left({ }^{1} \mathrm{H}\right)$ com z-gradient; obtiveram-se espectros com 32 e $64 \mathrm{~K}$ e largura de 12 e $31 \mathrm{kHz}$ para ${ }^{1} \mathrm{H} \mathrm{e}{ }^{13} \mathrm{C}$ em uma dimensão, respectivamente. Espectros $2 \mathrm{D}$ de correlação heteronuclear com detecção inversa foram coletados com $1 \mathrm{~K}$, largura de $5 \mathrm{kHz}$ em $F 2$, e $27 \mathrm{kHz}$ x 256 em F1. Os processamentos dos dados foram feitos com $1 \mathrm{~K}$ x $256 \mathrm{w}$, usando retorno linear em $F 1$ para gerar a matriz. Os valores de $J$ usados na implementação das sequências do HSQC e HMBC foram: $145 \mathrm{~Hz}$ $\left({ }^{1} \mathrm{~J}_{\mathrm{CH}}\right)$ e $9,0 \mathrm{~Hz}\left({ }^{\mathrm{n}} \mathrm{J}_{\mathrm{CH}}, \mathrm{n}>1\right)$, respectivamente.

Os experimentos de NOESY foram obtidos com $5 \mathrm{kHz}$ para $\mathrm{F} 1$ e F2 e blocos de $1 \mathrm{~K}$ x $1 \mathrm{~K}$, mixing time $1 \mathrm{~s}$, processados com ajustamento de fase, seguido de simetrização dos dados da matriz.

Processamentos semelhantes foram usados nos experimentos com o aparelho da Jeol, com parâmetros típicos para ${ }^{1} \mathrm{H}$ com pulso de $45^{\circ}$ (espectros com $16 \mathrm{~K}$ e com $15 \mathrm{ppm}$ ). Os experimentos 2D (HMQC e HMBC) foram modulados com os mesmos valores de ${ }^{1} \mathrm{~J}_{\mathrm{CH}} \mathrm{e}^{\mathrm{n}} \mathrm{J}_{\mathrm{CN}}$ 
descrito acima. Os experimentos para detectar NOE realizados no espectrômetro AC-200 foram obtidos com o programa NOEDIFF.AU da Bruker com irradiação na frequência das metoxilas.

Os espectros de massas de baixa resolução foram registrados em sistema acoplado CG/EM: Varian Saturn 2000 (3900GC e CP-1177); CG/EM HP-5989A: GC HP-5880A e HP-5897A com analisador de íons através de quadrupolo e ionização por impacto de elétrons, $70 \mathrm{eV}$.

Usaram-se placas de sílica gel $60 \mathrm{PF}_{254}$ Merck para cromatografia em camada delgada analítica e como reveladores utilizados luz ultravioleta (254 e $366 \mathrm{~nm}$ ), soluções de $\mathrm{AlCl}_{3}$-EtOH (1\%) e ácido difenilbórico etanolamina-MeOH (NP, 1\%).

\section{Material vegetal e isolamento dos flavonoides}

As partes aéreas de Solanum paludosum foram coletadas em janeiro de 1999 no campus universitário de João Pessoa, Paraíba e colocada em estufa a $40{ }^{\circ} \mathrm{C}$ por uma semana.

O material seco e pulverizado $(1,1 \mathrm{~kg})$ foi percolado com etanol. A solução extrativa foi concentrada em rotavapor à $40^{\circ} \mathrm{C}$ sob pressão reduzida, resultando em um extrato de cor verde escura $(100,1$ g). Este extrato foi dissolvido em ácido acético $20 \%$ e adicionado benzeno:éter, obtendo-se duas soluções extrativas, uma benzeno:éter etílico e outra ácida.

A solução extrativa benzeno:éter foi evaporada em rotavapor, fornecendo o extrato benzeno etéreo $(50,0 \mathrm{~g})$ que foi suspenso em metanol:água (8:2) e particionado com hexano e AcOEt. Os solventes hexano e AcOEt foram evaporados, fornecendo os extratos hexânicos $(31,5 \mathrm{~g})$ e acetato de etila $(12,0 \mathrm{~g})$.

$\mathrm{O}$ extrato hexânico foi solubilizado com $\mathrm{CH}_{2} \mathrm{Cl}_{2}$ e precipitado com $\mathrm{MeOH}$ a frio. $\mathrm{O}$ resíduo da água mãe foi submetido à cromatografia em coluna com sílica gel usando como eluentes hexano, AcOEt e $\mathrm{MeOH}$ em ordem crescente de polaridade, fornecendo doze frações. A quinta fração após recristalização rendeu 200,0 mg da substância $\mathbf{8}$, cristais amarelos em forma de agulhas. Este flavonoide foi metilado (20,0 mg) e acetilado originou 9 (18,0 mg; 90\%) e $\mathbf{1 0}$ (22,9 mg, rendimento quantitativo), respectivamente. A sexta fração foi submetida à cromatografia em coluna com sílica gel, tendo como eluentes hexano, acetato de etila e metanol, em grau crescente de polaridade, fornecendo quatro frações da qual a terceira fração cristalizou em forma de agulhas amarelas, substância $\mathbf{1 2}(25,0 \mathrm{mg})$ que foi acetilada (15,0 mg) fornecendo o flavonoide $\mathbf{1 3}(10,0 \mathrm{mg} ; 52,4 \%)$.

O resíduo obtido da solução AcOEt $(12,0 \mathrm{~g})$ foi cromatografado em Sephadex LH-20 (Coluna A), fornecendo quatro frações. A segunda fração (A-2) foi submetida à coluna rápida de sílica gel sob média pressão, coletando-se dez frações. As frações A-2/2 e A-2/3 forneceram um material cristalino amarelo, em forma de agulhas, que foram separados da água mãe e identificados como os flavonoides 11 (120,0 mg) e $\mathbf{1}$ (40,0 mg), respectivamente. O flavonoide 1 foi metilado $(4,0 \mathrm{mg})$ e acetilado $(10,0 \mathrm{mg})$ fornecendo a substância metilada 3 (4,0 mg; 95,7\%) e o flavonoide peracetilado 5 (10,0 mg; $70,4 \%)$, respectivamente. O constituinte $11(43,6 \mathrm{mg})$ também foi acetilado com anidrido acético na presença de piridina, fornecendo o produto $14(40,0 ; 58 \%)$. A água mãe da fração A-2/3 foi submetida a várias filtrações em colunas de Sephadex LH-20 e sílica gel sob média pressão, obtendo-se uma mistura contendo os flavonoides 1 e $\mathbf{1 5}(40,0 \mathrm{mg})$, o flavonoide $\mathbf{2}$ puro $(50,0 \mathrm{mg})$ e a mistura de $\mathbf{2}$ e $\mathbf{1 6}$ (19,8 mg). A terceira fração da coluna A (A-3) foi cromatografada em Sephadex LH-20, obtendo-se uma mistura das substâncias 7 e o ácido protocatecuico. O flavonoide $2(18,0 \mathrm{mg})$ foi acetilado e forneceu 4 (14,0 $\mathrm{mg} ; 61 \%)$.

As partes aéreas de Solanum jabrense foram coletadas na Paraíba, Nordeste do Brasil em setembro de 2000.
O pó seco e moído de $S$. jabrense (34 g) foi extraído com EtOH em temperatura ambiente até completa exaustão. A solução extrativa foi concentrada sob pressão reduzida fornecendo $5,3 \mathrm{~g}$ de $S$. jabrense. Uma parte do resíduo do extrato de $S$. jabrense foi suspenso em $\mathrm{H}_{2} \mathrm{O}: \mathrm{AcOH}$ (8:2) e extraído com hexano:éter (1:1). O solvente foi evaporado e o extrato hexano etéreo foi dissolvido em MeOH: $\mathrm{H}_{2} \mathrm{O}$ (4:1) e extraído com hexano e AcOEt. O extrato acetato de etila (1,2 g) foi cromatografado com Shephadex LH-20 em $\mathrm{CHCl}_{3}: \mathrm{MeOH}(1: 1)$ para remoção da clorofila dos flavonoides totais. As frações flavonoidicas obtidas foram analisadas em placas cromatográficas de camada fina com sílica gel 60 F254 e a visualização foi feita com a solução de difenilboriloxietilamina (NP, $1 \%$ ) em $\mathrm{MeOH}$ e $\mathrm{AlCl}_{3}$-EtOH (1\%), sendo observado na luz ultravioleta em $366 \mathrm{~nm}$. A fração foi filtrada com $\mathrm{CHCl}_{3}$ e $\mathrm{MeOH}$ em cartuchos de sílica C-18 (Sep-Pak C-18). A fração clorofórmica foi evaporada, redissolvida em $\mathrm{MeOH}$ e filtrada sobre a membrana de 0,45 $\mu \mathrm{m}$ (Millipore) para análise em CLAE.

A identificação das substâncias no extrato foi feita por comparação dos tempos de retenção entre os picos desconhecidos e os flavonoides padrões e por cocromatografia dos extratos com os flavonoides padrões. O cromatograma do extrato mostrou a presença de todos os flavonoides isolados de $S$. paludosum. Após a análise o extrato foi cromatografado com Sephadex LH-20 e foram isolados os flavonoides $\mathbf{1}, \mathbf{6}$ e 8.

Parte dos dados sobre o isolamento encontra-se registrados em publicações anteriores. ${ }^{1-3}$

\section{Preparação de derivados}

\section{Metilação com diazometano}

Uma solução de diazometano $\left(\mathrm{CH}_{2} \mathrm{~N}_{2}\right)$ foi preparada em éter e adicionada (em excesso) em gotas às soluções das substâncias em $\mathrm{CHCl}_{3}$ ou MeOH. Após a eliminação do solvente obtiveram-se as substâncias metiladas.

\section{Permetilação com sulfato de dimetila}

Sulfato de dimetila $\left(\mathrm{Me}_{2} \mathrm{SO}_{4}\right)$ foi usado para a permetilação dos flavonois. À solução dos flavonoides em acetona seca adicionou-se 1,1 eq. de $\mathrm{K}_{2} \mathrm{CO}_{3}$, e 1,1 eq. de sulfato de dimetila para cada hidroxila livre. A mistura resultante, na temperatura ambiente, foi agitada durante 2 h e monitorada através de análise por placa cromatográfica. Após a conclusão da reação, o solvente foi destilado em rotavapor sob pressão reduzida e o material oleoso obtido foi suspenso em água (cerca de $50 \mathrm{~mL}$ ), tratado com $5 \mathrm{~mL}$ de solução de hidróxido de amônio concentrado e extraído com $\mathrm{CH}_{2} \mathrm{Cl}_{2}$ (com 3 vezes $15 \mathrm{~mL}$ ). A solução das frações orgânicas reunidas foi seca com sulfato de sódio anidro e o solvente removido em rotavapor mantido sob pressão reduzida. Em alguns casos obteve-se recristalização em acetona ou $\mathrm{MeOH}{ }^{4}$

As substâncias foram submetidas a tratamento com anidrido acético e piridina (1:1) e a mistura mantida em repouso durante 48 h. Após esse período, foi tratada com água destilada gelada, obtendose o material precipitado. O precipitado foi filtrado e lavado várias vezes com água destilada, dissolvido em diclorometano e seco com sulfato de sódio anidro. Após evaporação do solvente em rotavapor obtiveram-se as substâncias acetiladas.

\section{RESULTADOS E DISCUSSÃO}

Os flavonoides $(\mathbf{1}, \mathbf{2}, \mathbf{7}, \mathbf{8}, \mathbf{1 1}, \mathbf{1 2}, \mathbf{1 5}$ e 16) foram isolados das partes aéreas de $S$. paludosum através de cromatografia utilizando Sephadex LH-20. ${ }^{1,2}$ A metilação do flavonoide 1 com diazometano forneceu 3, contendo grupo metoxila adicional na posição 4', enquan- 
to que a acetilação de $\mathbf{2}$ produziu $\mathbf{4}$, derivado acetilado com funções acetoxílicas nas posições 3 e 4' e de $\mathbf{1}$ forneceu $\mathbf{5}$, sustentando grupos acetoxílicos nas posições 3,5 e 4'. O flavonoide 8 foi metilado com sulfato de dimetila e acetilado com anidrido acético na presença de piridina para fornecer os derivados permetilado 9 e o derivado monoacetílico 10 (acetila na posição 5), respectivamente. Os flavonóides 11 e 12 foram acetilados com anidrido acético na presença de piridina e forneceram os produtos acetilados 14 e 13, respectivamente.

Das partes aéreas de $S$. jabrense foram isolados os flavonoides $\mathbf{1}$, $\mathbf{6}$ e 8, sendo os demais $(\mathbf{2}, \mathbf{7}, \mathbf{1 1}$ e 12) identificados nos tricomas das partes aéreas através de análise por cromatografia de alta eficiência (CLAE). ${ }^{3}$

Os padrões de substituição dos anéis aromáticos (anéis A e B) e heterocíclico (anel C) dos flavonoides isolados foram usados para definir os grupos responsáveis pelos diferentes perfis observados nos espectros de $\mathrm{RMN}^{1} \mathrm{He}^{13} \mathrm{C}$. A Figura 1 revela as estruturas dos flavonoides naturais e dos derivados obtidos por metilação e/ou acetilação.

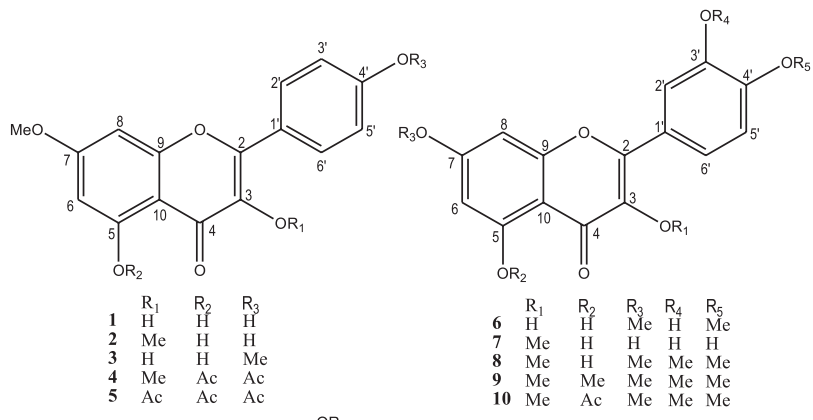<smiles>COc1ccc(-c2oc3c(OC)c(OC)cc(O)c3c(=O)c2O)cc1Oc1cc(O)c2c(=O)cc(-c3ccc(O)cc3)oc2c1</smiles>

Figura 1. Estrutura dos flavonoides naturais e dos derivados obtidos por metilação e acetilação

\section{Espectro de RMN $^{1} \mathrm{H}$ dos flavonoides}

\section{Caracterização do anel A}

O padrão de oxigenação dos flavonoides naturais $(\mathbf{1}, \mathbf{2}, \mathbf{6 - 8}, \mathbf{1 1}$ $12,15$ e 16$)$ e dos derivados $(3-5,9-10,13$ e 14) foi determinado pelo espectro de RMN ${ }^{1} \mathrm{H}$ (Tabela 1). Os espectros dos flavonóis 11-14 e 16 apresentaram apenas um sinal simples com deslocamento químico entre $\delta_{\mathrm{H}} 6,41$ e 6,66 para um hidrogênio aromático localizado no anel A, indicando a presença de três substituintes oxigenados. A presença de um simpleto em região mais desblindada dos flavonóis naturais 11 e $12\left(\delta_{\mathrm{H}} 12,47\right)$ e $\mathbf{1 6}\left(\delta_{\mathrm{H}} 12,55\right)$ indicou a presença de uma hidroxila quelatogênica em C-5. A acetilação da hidroxila quelada HO-5 em 13 e 14 gerou, como previsto, a desblindagem $(\Delta \delta=0,25$ ppm) no hidrogênio H-6 presente no anel A em posição orto, ${ }^{5}$ como consequência da atenuação do efeito mesomérico promovido pela deslocalização dos elétrons não compartilhados do átomo de oxigênio devido à presença da carbonila do grupo acetoxila.
Os espectros dos flavonóis 1-10 e da flavona $\mathbf{1 5}$ revelaram a presença de dois sinais para átomos de hidrogênio localizados no anel A (Tabela 1), com a constante acoplamento $(J)$ indicando interação spin-spin de hidrogênios mantendo entre si relação meta $(J=1,9-2,2$ $\mathrm{Hz})$ e correspondendo aos hidrogênios H-6 $\left(\delta_{\mathrm{H}} 6,25-6,66\right)$ e H-8 $\left(\delta_{\mathrm{H}} 6,46-6,84\right)$.

\section{Caracterização do anel $B$}

$\mathrm{O}$ anel B dos flavonóis 1-5, 15 e $\mathbf{1 6}$ revelou a presença de sinais característicos de um sistema aromático para dissubstituído, sendo, consequentemente, representado por dois dupletos correspondentes aos dois pares de átomos de hidrogênio (H-2'/H-6': $\delta_{\mathrm{H}} 7,85-8,11$; e H-3'/H-5' : $\left.\delta_{\mathrm{H}} 6,67-7,25\right)$ acoplados através de interação spin-spin em orto $(J=6,0-8,8 \mathrm{~Hz})$, constituindo um típico sistema AA'BB' (Tabela 1).

O anel B dos flavonóis 6-14 apareceu representado por três sinais (Tabela 1) correspondentes aos três hidrogênios localizados em ambientes químicos diferentes $\left(\mathrm{H}_{-2} 2^{\prime}: \delta_{\mathrm{H}} 7,67-7,91 ; \mathrm{H}-5\right.$ ' : $\delta_{\mathrm{H}} 6,98-7,10 ; \mathrm{H}-6$ ': $\left.\delta_{\mathrm{H}} 6,69-8,08\right)$. Os valores dos deslocamentos químicos e das constantes de acoplamento (meta: $J=1,3-2,4 \mathrm{~Hz}$; orto: $J=8,4-8,8 \mathrm{~Hz}$ ) destes átomos de hidrogênio (Tabela 1) permitiram definir o padrão de substituição do anel B. Um dos sinais apareceu como um duplo dupleto (em alguns como um simpleto largo) acoplando com os dois hidrogênios restantes que não acoplam entre si. Os valores das constantes de acoplamento $(J=1,3-2,4$ e 8,4-8,8 Hz) são característicos de interações meta e orto também em flavonoides oxigenados. Os valores dos deslocamentos químicos e das constantes de acoplamento observadas nos espectros de $\mathrm{RMN}^{1} \mathrm{H}$ dos flavonóis 6-14 permitiram propor a localização das funções oxigenadas nas posições 3' e 4' (Tabela 1).

\section{Caracterização do anel $C$}

Apenas o flavonoide $\mathbf{1 5}$ apresentou um sinal simpleto em $\delta_{\mathrm{H}} 6,82$, sugerindo a presença de um hidrogênio metínico, H-3, característico de flavona. ${ }^{6}$

Além do padrão de substituição dos anéis presentes no esqueleto de flavonoides, os espectros de $\mathrm{RMN}{ }^{1} \mathrm{H}$ permitiram deduzir a natureza e o número de grupos substituintes (sinais simples de grupos metoxílicos $\delta_{\mathrm{H}} \cdot 3,84$ - 3,96; grupos hidroxílicos localizados em C-5, envolvido em ligação de hidrogênio $\delta_{\mathrm{H}} 12,13$ - 12.94 e, as hidroxilas localizadas em outras posições revelaram-se mais sensíveis aos efeitos de solvente, $\mathrm{H}_{2} \mathrm{O}$ presente no solvente e temperatura. Os espectros de $\mathrm{RMN}^{1} \mathrm{H}$ dos derivados acetilados, 4, 5, 10, 13 e 14 mostraram também a presença de sinais simples entre $\delta_{\mathrm{H}}$ 2.3-2.5 referentes aos grupos metílicos das acetoxilas (Tabela 1).

\section{Análise dos dados de massas}

Os espectros de massas dos flavonoides naturais mostraram picos intensos correspondentes aos íons moleculares: $\mathbf{1}(\mathrm{m} / \mathrm{z}, 300$, $\left.\left[\mathbf{M}^{+}\right]\right), \mathbf{2}\left(\mathrm{m} / \mathrm{z}, 314,\left[\mathbf{M}^{+}\right]\right), 7\left(\mathrm{~m} / \mathrm{z}, 316,\left[\mathbf{M}^{+}\right]\right), \mathbf{8}\left(\mathrm{m} / \mathrm{z}, 358,\left[\mathbf{M}^{+}\right]\right), \mathbf{1 1}$ $\left(\mathrm{m} / \mathrm{z}, 374,\left[\mathbf{M}^{+}\right]\right), \mathbf{1 2}\left(\mathrm{m} / \mathrm{z}, 388,\left[\mathbf{M}^{+}\right]\right)$e $\mathbf{1 6}\left(\mathrm{m} / \mathrm{z}, 344\left[\mathrm{M}^{+}\right]\right)$. Com base no pico correspondente ao íon molecular pôde-se deduzir o número de hidroxilas e metoxilas presentes como substituintes. O flavonoide acetilado, 4 não revelou o pico correspondente ao íon molecular $(\mathrm{m} / \mathrm{z}$, 356) e mostrou o pico em $\mathrm{M}^{+}-42\left(\left[\mathrm{M}-\mathrm{CH}_{2}=\mathrm{C}=\mathrm{O}\right]^{+}\right)$devido à fragmentação do grupo acetoxila com eliminação de ceteno $\left(\mathrm{O}=\mathrm{C}=\mathrm{CH}_{2}\right)$. Os derivados acetilados $\mathbf{5}\left(\mathrm{m} / \mathrm{z}, 426,\left[\mathbf{M}^{+}\right]\right) \mathbf{1 3}\left(\mathrm{m} / \mathrm{z}, 388,\left[\mathbf{M}^{+}\right]\right)$e $\mathbf{1 4}$ $\left(\mathrm{m} / \mathrm{z}, 416,\left[\mathbf{M}^{+}\right]\right)$revelaram os picos referentes aos íons moleculares. Os espectros de massas dos flavonoides naturais $11(\mathrm{~m} / \mathrm{z}, 359), 12$ $(\mathrm{m} / \mathrm{z}, 373)$ e $\mathbf{1 6}(\mathrm{m} / \mathrm{z}, 329)$ sustentando uma metoxila em C-8 revelaram pico base em [M-15] $]^{+}$e o derivado acetilado $13(\mathrm{~m} / \mathrm{z}, 359) \mathrm{em}$ ([M-42-15 $]^{+}$), em acordo com Goudard et al., ${ }^{7}$ que sugeriu a relação entre as intensidades $[\mathrm{M}]^{+} /[\mathrm{M}-15]^{+}$como um método para distinguir 


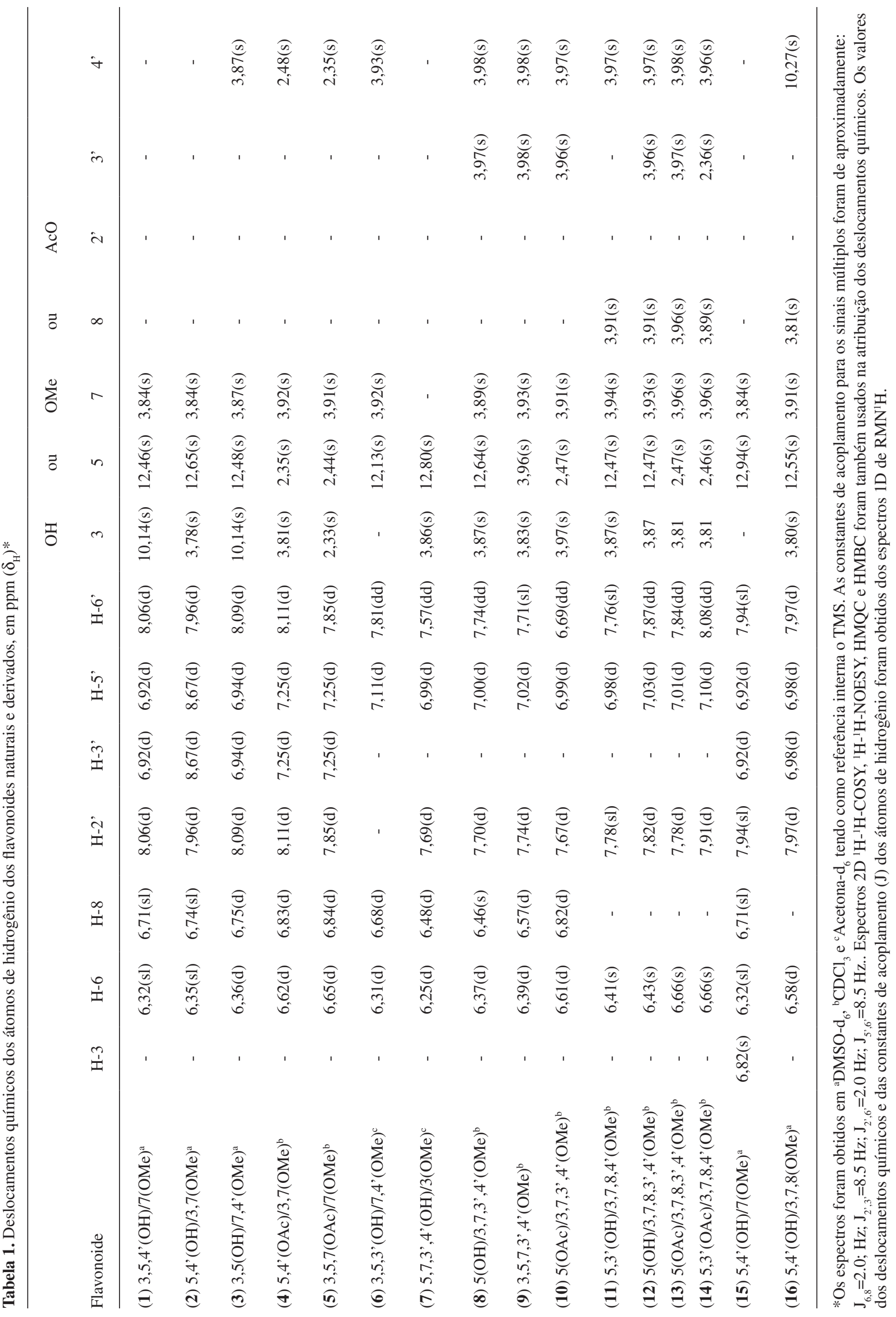


os flavonoides com os substituintes nas posições 5,6,7 ou 5,7 e 8. A abundância relativa dos picos M e M-15 no espectro de massas permite a diferenciação de 5-hidroxi-7,8-dimetoxi e 5,7,8-trimetoxiflavonas $(\mathrm{M}>\mathrm{M}-15)$ de 5-hidroxi-7,8-dimetoxi e 5,6,7-trimetoxiflavonas $(\mathrm{M}<\mathrm{M}-15)$, respectivamente.

A perda de um radical hidrogênio a partir do íon molecular, originando um pico com abundância relativa maior que $50 \%$, foi observada nos espectros de massas dos flavonoides que não sustentam metoxila em C-8 1-3, 6 - 9 e 15 e nos derivados acetilados 4 , $5,10,13$ e 14. A preferência para a eliminação de H-2' e/ou H-6' pode ser justificada pela formação de íon com estabilidade justificada por efeito mesomérico (I), o mesmo não ocorrendo com II formado pela eliminação de radical do flavonoide $\mathbf{1 5}$. Nos casos da presença de grupo metoxila em C-8 ocorre influência favorável à reação de eliminação de radical metila (III). O mesmo podendo ocorrer com flavonoides sustentando metoxila em C-6 (IV) (Figura 2).<smiles>[2H]Oc1c(C)oc2c(=O)c(C)c(C)oc12</smiles><smiles>COC1CCC2OC(=C3C=CC(=O)C=C3)C=C(O)C12</smiles><smiles>CCCCC1CCC(=O)OC1=O</smiles>

III<smiles>[2H]C1=CC2=CCCCC2C(=O)C1=O</smiles>

IV

Figura 2. Íons de flavonoides formados por: efeito mesomérico (I), eliminação de radical do flavonoide 15 (II), presença do grupo metoxila em $C-8$ (III) e em C-6 (IV)

Outros picos representantes de íons formados a partir do íon molecular podem ser justificados pela eliminação de unidade estrutural representada pela perda de $30\left(\mathrm{H}_{2} \mathrm{CO}\right), 29(\mathrm{HCO}), 18\left(\mathrm{H}_{2} \mathrm{O}\right)$, $43\left(\mathrm{CH}_{3} \mathrm{CO}\right)$, que foram observados em vários espectros de massas como unidades de massa/carga.

A fragmentação mais característica de todos os flavonoides foi atribuída à clivagem no anel $\mathrm{C}$ pela reação retro Diels-Alder, ${ }^{8}$ fornecendo os fragmentos representados por $\mathbf{V}$ e VI com valores de $\mathrm{m} / \mathrm{z}$ dependentes dos substituintes presentes em cada um dos anéis A e B (Figura 3). Sendo R=OMe (VIb),observado em flavonoides com metoxila em C-3, verifica-se condição estrutural para eliminar formaldeido $\left(\mathrm{CH}_{2} \mathrm{O}=30\right)$ e fornecer também o fragmento representado por VIa (Tabela 2).

Tais fragmentos podem ser acompanhados por VII $(\mathbf{V I}+\mathrm{H}$ oriundo de rearranjo) e VIII formado através de processo de fragmentação que preserva a ligação do oxigênio 1 e o átomo de carbono C-2 (Tabela 2).

Nos casos de 1, 2, 4-14 e 16 aparecem os picos com valores típicos para os padrões de substituição (Ia-Ic; IIa-IId) nos aneis A e B (Figura 4 e Tabela 2).

\section{Análise dos dados de $\mathrm{RMN}{ }^{13} \mathrm{C}$}

Embora as informações obtidas nos espectros de $\mathrm{RMN}^{1} \mathrm{H}$ e de massas tenham permitido deduzir as propostas das estruturas, infor-
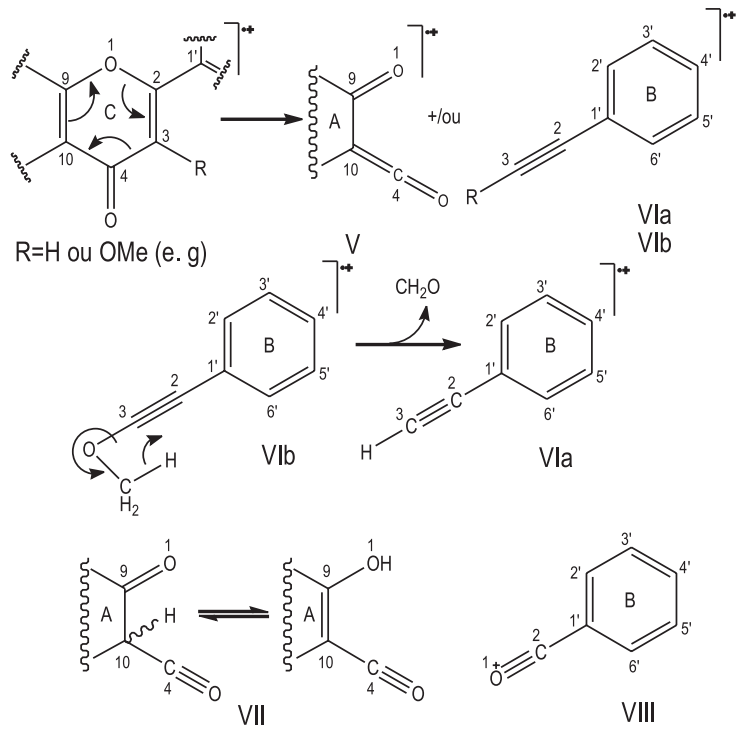

Figura 3. Fragmentação de retro Diels-Alder<smiles>[R6]OC1=CC([R])=CC(=O)C1C#N</smiles>

la $\mathrm{R}=\mathrm{H} \quad \mathrm{m} / \mathrm{z} 153$

Ib R=Me, $\mathrm{R}_{1}=\mathrm{H} \mathrm{m} / \mathrm{z} 167$

Ic $\mathrm{R}=\mathrm{R} 1=\mathrm{Me} \quad \mathrm{m} / \mathrm{z} 181$<smiles>[R]Oc1ccc(C#[N+])cc1[R]</smiles>

Ila $\mathrm{R}=\mathrm{R}_{1}=\mathrm{H} \quad \mathrm{m} / \mathrm{z} 121$ Ilb $\mathrm{R}=\mathrm{H}, \mathrm{R}_{1}=\mathrm{OH} \quad \mathrm{m} / \mathrm{z} 137$ Ilc $\mathrm{R}=\mathrm{Me}, \mathrm{R}_{1}=\mathrm{OH} \quad \mathrm{m} / \mathrm{z} 151$ Ild $\mathrm{R}=\mathrm{Me}, \mathrm{R}_{1}=\mathrm{OMe} \mathrm{m} / \mathrm{z} 165$
Figura 4. Estruturas dos picos principais observados nos espectros de massas dos flavonoides 1, 2, 4-14 e 16

mações relevantes e mais conclusivas foram obtidas pelos espectros de $\mathrm{RMN}{ }^{13} \mathrm{C}$. Os dados obtidos nos espectros de RMN ${ }^{13} \mathrm{C}$ dos flavonoides naturais e dos derivados metilado e acetilado encontram-se na Tabela 2.

\section{Sinais de carbonos metoxílicos}

É relativamente bem conhecido ${ }^{7-10}$ que os sinais de carbonos metoxílicos localizados em sistemas aromáticos envolvidos em impedimento estérico aparecem em torno de $\delta_{\mathrm{CH} 3} 60$, revelando diferença significativa quando se compara com valores em torno de $\delta_{\mathrm{CH} 3} 56$ observados quando se encontram em posições livres de influência estérica (sem substituintes nas duas posições orto). A combinação das informações fornecidas pelos espectros de $\mathrm{RMN}{ }^{1} \mathrm{H}$ sobre o padrão de substituição e os deslocamentos químicos dos carbonos de grupos metoxílicos revelados pelos espectros de $\mathrm{RMN}{ }^{13} \mathrm{C}$ permitiu eliminar muitos isômeros estruturais, simplificando os trabalhos de elucidação estrutural. No caso de 7 (3-O-metilquercetina), o simples sinal da única metoxila em $\delta_{\mathrm{CH} 3} 58.84$ revelou-se consistente somente com a metoxilação no C-3. De modo semelhante, o flavonol 11 mostrou a presença de dois sinais com deslocamentos químicos característicos de duas metoxilas impedidas, permitindo admitir a presença de $\mathrm{MeO}$ 3 e em mais uma posição com impedimento estérico. Nos casos em que aparecem sinais de metoxilas em $\approx \delta_{\mathrm{CH} 3} 56.00$ podem-se descartar as possibilidades de localização de grupo metoxila em C-3 ou em outra posição impedida, como foi observado para as substâncias naturais 1,3 e 15, que permitiram definir a metoxilação somente nas posições, C-7 ou C-4' (Tabela 3). 
Tabela 2. Picos principais observados nos espectros de massas dos flavonoides $\mathbf{1 , 2 , 4 - 1 4}$ e 16

Flavonoide

(1) $3,5,4^{\prime}(\mathrm{OH})_{3} / 7(\mathrm{OMe})$

(2) $5,4^{\prime}(\mathrm{OH})_{2} / 3,7(\mathrm{OMe})_{2}$

(4) $5,4^{\prime}(\mathrm{OAc})_{2} / 3,7(\mathrm{OMe})_{2}$

(5) $3,5,4$ '(OAc) $)_{3} / 7(\mathrm{OMe})$

(6) $3,5,3^{\prime}(\mathrm{OH})_{3} / 7,4^{\prime}(\mathrm{OMe})_{2}$

(7) $5,7,3^{\prime}, 4^{\prime}(\mathrm{OH})_{4} / 3(\mathrm{OMe})$

(8) $5(\mathrm{OH}) / 3,7,3^{\prime}, 4^{\prime}(\mathrm{OMe})_{4}$

(9) $3,5,7,3^{\prime}, 4^{\prime}(\mathrm{OMe})_{5}$

(10) $5(\mathrm{OAc}) / 3,7,3^{\prime}, 4^{\prime}-(\mathrm{OMe})_{4}$

(11) $5,3^{\prime}(\mathrm{OH})_{2} / 3,7,8,4^{\prime}(\mathrm{OMe})_{4}$

(12) $5(\mathrm{OH}) / 3,7,8,3^{\prime}, 4^{\prime}(\mathrm{OMe})_{5}$

(13) $5(\mathrm{OAc}) / 3,7,8,3^{\prime}, 4^{\prime}(\mathrm{OMe})_{5}$

(14) $5,3^{\prime}(\mathrm{OAc})_{2} / 3,7,8,4^{\prime}(\mathrm{OMe})_{4}$

(16) $5,4^{\prime}(\mathrm{OH})_{2} / 3,7,8(\mathrm{OMe})_{3}$
$\operatorname{Picos}(m / z)$
$300\left([\mathrm{M}]^{+}, 100 \%\right), 167$ (Ib, 6\%), 121 (IIa, 26\%)

$314\left([\mathrm{M}]^{+}, 100 \%\right), 296\left(\mathrm{M}-\mathrm{H}_{2} \mathrm{O}, 15 \%\right), 167$ (Ib 11\%), 121 (IIa, 40\%)

$314\left(\mathrm{M}-\mathrm{CH}_{2}=\mathrm{C}=\mathrm{O}-\mathrm{CH}_{2}=\mathrm{C}=\mathrm{O} 100 \%\right), 296\left(\mathrm{M}-\mathrm{H}_{2} \mathrm{O}, 15 \%\right), 167$ (Ib, 11\%), 121(IIa, após eliminação de $\mathrm{CH}_{2}=\mathrm{C}=\mathrm{O}$ e $\mathrm{CH}_{2}=\mathrm{O}, 40 \%$ )

$426\left([\mathrm{M}]^{+}, 2 \%\right), 342\left(\mathrm{M}-\mathrm{CH}_{2}=\mathrm{C}=\mathrm{O}-\mathrm{CH}_{2}=\mathrm{C}=\mathrm{O}, 22 \%\right), 300\left(\mathrm{M}-\mathrm{CH}_{2}=\mathrm{C}=\mathrm{O}-\mathrm{CH}_{2}=\mathrm{C}=\mathrm{O}-\right.$ $\left.\mathrm{CH}_{2}=\mathrm{C}=\mathrm{O}, 100 \%\right), 121($ IIa, 10\%)

$330\left([\mathrm{M}]^{++}, 7 \%\right.$ ), $300\left(\mathrm{M}-\mathrm{H}_{2} \mathrm{C}=\mathrm{O}, 100 \%\right), 167$ (Ib, 6\%), 121 (IIa, após eliminação de $\mathrm{H}_{2} \mathrm{C}=\mathrm{O}$, $26 \%)$

$316\left([\mathrm{M}]^{++}, 100 \%\right), 301(\mathrm{M}-\mathrm{Me}, 22 \%), 137(\mathbf{I I b}, 43 \%)$

$358\left([\mathrm{M}]^{+}, 100 \%\right), 327\left(\mathrm{M}-\mathrm{H}-\mathrm{H}_{2} \mathrm{C}=\mathrm{O}, 12 \%\right), 343\left(\mathrm{M}-\mathrm{CH}_{3}, 42 \%\right), 315\left(\mathrm{M}-\mathrm{CH}_{3}-\mathrm{C}=\mathrm{O}\right.$, $38 \%$ ), 167 (Ib, 5\%), 165 (IId, 18\%), 135 (IId - $\left.\mathrm{H}_{2} \mathrm{C}=\mathrm{O}, 5 \%\right)$

$372\left([\mathrm{M}]^{+}, 100 \%\right), 357\left(\mathrm{M}-\mathrm{CH}_{3}, 70 \%\right), 341\left(\mathrm{M}-\mathrm{H}-\mathrm{H}_{2} \mathrm{C}=\mathrm{O}, 20 \%\right), 311\left(\mathrm{M}-\mathrm{CH}_{2}=\mathrm{C}=\mathrm{O}-\right.$ $\left.\mathrm{CH}_{2}=\mathrm{C}=\mathrm{O}, 14 \%\right), 181($ Ic, $7 \%), 165($ IId, 11\%)

$358(\mathrm{M}-42,100 \%), 343\left(\mathrm{M}-\mathrm{CH}_{3}, 42 \%\right), 315\left(\mathrm{M}-\mathrm{CH}_{3}-\mathrm{C}=\mathrm{O}, 38 \%\right), 167$ (Ib, após eliminação de $\left.\mathrm{CH}_{2}=\mathrm{C}=\mathrm{O}, 5 \%\right), 165$ (IId, 18\%), $135\left(\right.$ IId $\left.-\mathrm{H}_{2} \mathrm{C}=\mathrm{O}, 5 \%\right)$

$374\left([\mathrm{M}]^{++}, 86 \%\right), 359(\mathrm{M}-\mathrm{Me}, 100 \%), 151$ (IIc, 10\%)

$388\left([\mathrm{M}]^{+}, 7 \%\right), 345(\mathrm{M}-\mathrm{Me}-\mathrm{C}=\mathrm{O}, 4 \%), 373(\mathrm{M}-\mathrm{Me}, 100 \%), 165$ (IId, 9\%)

$388\left(\mathrm{M}-\mathrm{CH}_{2}=\mathrm{C}=\mathrm{O}, 64 \%\right), 373(\mathrm{M}-\mathrm{Me}, 100 \%), 345(\mathrm{M}-\mathrm{Me}-\mathrm{C}=\mathrm{O}, 5 \%), 165($ IId, 9\%)

416 (100\%), 401 (M - Me, 98\%), $359\left(\mathrm{CH}_{2}=\mathrm{C}=\mathrm{O}-\mathrm{Me}, 95 \%\right) 151$ (IIc, após eliminação de $\mathrm{CH}_{2}=\mathrm{C}=\mathrm{O}, 8 \%$ )

$344\left([\mathrm{M}]^{+}, 49 \%\right), 329(\mathrm{M}-\mathrm{Me}, 100 \%), 314$ (M - Me -Me, 19\%) 121 (IIa, 23\%)
Distinção entre flavonois e flavonas com base nos sinais dos carbonos do anel $C$

A distinção entre flavonas (H-3) e flavonois (RO-3) foi verificada pela ausência do sinal de carbono metínico em torno de $\delta_{\mathrm{C}}$ 103 (CH-3 de flavonas) e a presença do sinal de C-3 em torno $\delta_{\mathrm{C}}$ 136-140 (C-3, carbono não hidrogenado de flavonóis). O maior deslocamento químico do C-3 oxigenado justifica-se pelo efeito indutivo retirador de elétrons do átomo de oxigênio, que exerce efeito protetor por mesomeria sobre o átomo de carbono C-2. ${ }^{9,10}$ $\mathrm{O}$ sinal de carbono $\mathrm{C}-3$ oxigenado desloca-se para região mais desblindada $\left(\Delta \delta_{\mathrm{C}}=10 \mathrm{ppm}\right)$ e o do $\mathrm{C}-2$ para região mais blindada por cerca de $\Delta \delta_{\mathrm{C}}=17$ ppm quando comparados com os valores correspondentes de uma flavona. O deslocamento químico do sinal da carbonila é influenciado pela interação da ligação intramolecular com o hidrogênio do grupo HO-3 e HO-5. O primeiro causando uma blindagem de cerca de $\Delta \delta_{C}=5 \mathrm{ppm}$ e o último uma desblindagem de magnitude similar. Assim, flavonas sem grupo hidroxila em C-3 e 5-hidroxiflavonóis apresentam sinais praticamente com o mesmo deslocamento químico para a carbonila, já que os efeitos acima referidos para as hidroxilas se anulam. A metilação de HO-5 elimina o efeito da ligação de hidrogênio sobre o C-4 e, consequentemente, os flavonóis com H-5 ou MeO-5 apresentam aproximadamente o mesmo deslocamento químico para a carbonila C-4. A metilação do grupo HO-3 somente reduz parcialmente este efeito sobre C-4, como pode ser visto quando comparamos o flavonol $1, \delta_{\mathrm{C}-4} 176,03$
(HO-3) com o $2 \delta_{\mathrm{C}-4}$ 178,09 (MeO-3). Embora o sinal da carbonila possa fornecer informação quanto à substituição em C-5, a distinção entre flavonóis e seus 3-metoxi-derivados foi baseada nos deslocamentos químicos de C-2 e C-3. Comparando-se os dados de 1 com 2 foi possível deduzir claramente que a metilação do HO-3 provocou uma desblindagem de $\Delta \delta_{\mathrm{C}}=8,7$ e 1,9 ppm nos sinais de $\mathrm{C}-2$ e C-3, respectivamente. Um pequeno deslocamento nos sinais de $\mathrm{C}-10(+1,1)$ e nos dois carbonos do anel $\mathrm{C}, \mathrm{C}-1^{\prime}\left(\Delta \delta_{\mathrm{C}}=-1,1\right)$ e C-4' $\left(\Delta \delta_{C}=+0,96\right)$ foi observado pela metilação de HO-3. ${ }^{11}$ Do mesmo modo, através da comparação dos dados da flavona 15 e do flavonol 1 observou-se que a troca de $\mathrm{H}-3$ por hidroxila causou modificação nos deslocamentos químicos de C-4 $\left(\Delta \delta_{C}=+5,9 \mathrm{ppm}\right)$, C-2 $\left(\Delta \delta_{\mathrm{C}}=-16,8 \mathrm{ppm}\right)$ e C-4' $\left(\Delta \delta_{\mathrm{C}}=-1,9 \mathrm{ppm}\right)$.

\section{Caracterização do anel B}

Anel B mono-oxigenado

Os sinais dos carbonos do anel B dos flavonóis $\mathbf{1}$ - 5, 15 e 16 foram facilmente assinalados, usando-se a influência dos efeitos dos substituintes nos deslocamentos químicos e a simetria promovida pela presença de um único substituinte oxigenado no anel aromático. Dois sinais no espectro de $\mathrm{RMN}{ }^{13} \mathrm{C}$ representando dois pares de carbonos metínicos e os correspondentes deslocamentos químicos indicaram a presença de função oxigenada localizada em C-4'. O maior deslocamento químico dos sinais metínicos (em torno de $\delta_{\mathrm{C}}$ 


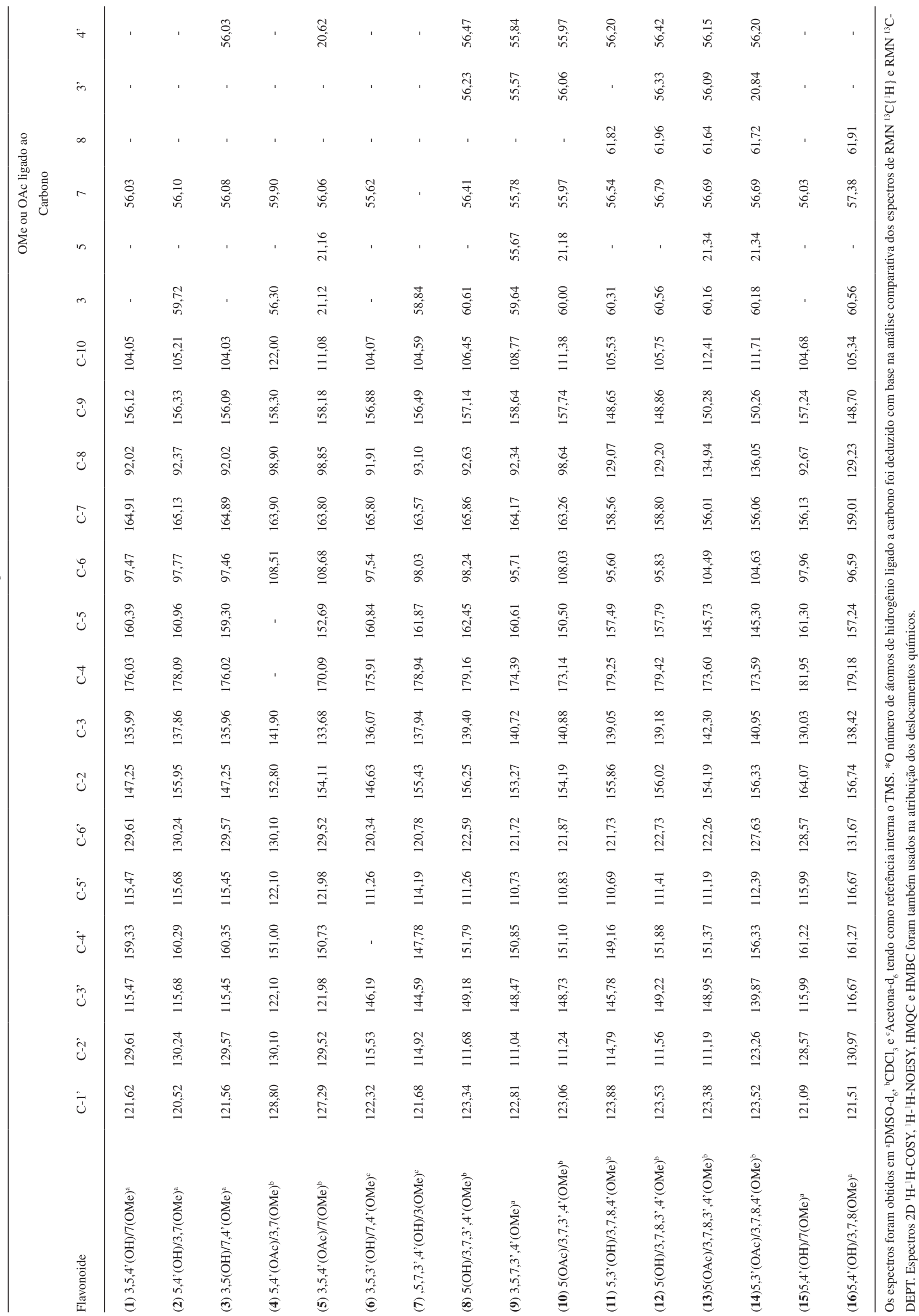


130) é aproximadamente o mesmo deslocamento químico encontrado para o benzeno, revelando que estes carbonos ocupam posição meta em relação à oxigenação, ${ }^{12}$ sendo, portanto, atribuído aos $\mathrm{CH}-2$ ' e CH-6'. O deslocamento químico do outro sinal ( $\left.\mathrm{CH}^{-} 3^{\prime}, \mathrm{CH}-5^{\prime}\right)$ é típico de carbonos localizados em posição orto em relação a uma função oxigenada, cuja blindagem mesomérica desloca o sinal para região mais blindada (quando comparado ao benzeno) para cerca de $\delta_{\mathrm{C}} 115$ (flavonóides 1, 2 e 15).

As substâncias $\mathbf{1}$ e $\mathbf{2}$ após a acetilação da $\mathrm{HO}$ em C-4' forneceram os derivados 5 e 4, respectivamente, que apresentaram mudanças nos deslocamentos químicos correspondentes aos carbonos ipso (blindagem $\Delta \delta_{\mathrm{C}}=-9 \mathrm{ppm}$ ), orto (desblindagem, $\Delta \delta_{\mathrm{C}}=+6 \mathrm{ppm}$ ) e para (desblindagem $\Delta \delta_{C}=+8 \mathrm{ppm}$ ) como esperado. ${ }^{7}$ Nestes valores inclui-se o efeito de solvente, já que, segundo Pelter et $a l,{ }^{13}$ nos espectros dos flavonoides o valor do deslocamento químico dos carbonos individualmente aumenta por cerca de $0,5 \mathrm{ppm}$ quando se troca o solvente o $\mathrm{CDCl}_{3}$ por DMSO- $d_{6}$.

\section{Caracterização de anel B 3',4'-dioxigenado}

Os flavonóis desta categoria puderam ser apresentados em três grupos: um apresentando duas hidroxilas, 7 [ $\delta_{\mathrm{C}}$ : 114,92 (CH-2'), 114,19 (CH-5') e 120,78 (CH-6')]; dois representam uma hidroxila e uma metoxila, $6\left[\delta_{\mathrm{C}}\right.$ : 115,53 (CH-2'), 111,26 (CH-5') e 120,34

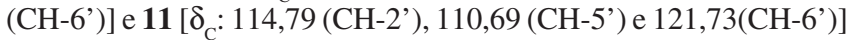
e cinco flavonoides com duas metoxilas, 8-10 [ $\delta_{\mathrm{C}}: 111,04-111,68$ (CH-2'), 110,73 - 11,26 (CH-5') e 121,72 - 122,59(CH-6')], 12 [ $\delta_{\mathrm{C}}$ : 111,58 (CH-2'), 111,41 (CH-5') e 122,73 (CH-6')] e 13 [ $\delta_{\mathrm{C}^{\prime}}: 111,19$ (CH-2'), 111,19 (CH-5') e 122,26 (CH-6')]. Os produtos de acetilação foram também considerados para confirmações estruturais. A comparação destes deslocamentos químicos permitiu também avaliar os efeitos da conversão de uma hidroxila em metoxila e em acetoxila. A localização das funções oxigenadas em carbonos adjacentes tornou-se relativamente facilitada pelos valores dos deslocamentos químicos em torno de $\delta_{\mathrm{C}} 146$ (C-3') e 151 (C-4', posição conjugada com o grupo carbonila e, consequentemente, maior deslocamento químico devido ao efeito mesomérico retirador de elétrons da carbonila) exibindo um efeito de blindagem de $\Delta \delta_{C}=10 \mathrm{ppm}$ que cada oxigênio exerce no carbono orto. A posição certa da oxigenação dos flavonóis 6 e 7 foi determinada através da comparação com os modelos I, II e III (Figura 5). ${ }^{14} \mathrm{O}$ flavonol 11 apresentou o anel B com o mesmo padrão de oxigenação de $\mathbf{6}$, mas os espectros foram obtidos em $\mathrm{CDCl}_{3} \mathrm{e}$ acetona- $d_{6}(6$ e 7) e, por isso, tornou-se necessário a utilização de comentários adicionais.

Na unidade parcial do modelo III (em relação a I, com anel B semelhante ao de 7) e no flavonol 6 a troca da hidroxila em C-4' por uma metoxila produziu um deslocamento para região mais blindada (menor deslocamento químico) de aproximadamente $\Delta \delta_{C}=-4,0 \mathrm{ppm}$ no carbono 5' (pode ser atribuído a efeito $\gamma$ do carbono metoxílico), localizado em posição orto. O C-1' localizado em posição para apresentou uma desblindagem de $\Delta \delta_{\mathrm{C}}=+1,0$. Os carbonos C-2' e C-6' estão dispostos simetricamente em relação a um eixo imaginário contendo C-1' e C-4', sendo a diferença dos deslocamentos químicos dos seus sinais atribuída à presença do substituinte oxigenado em C-3'. Devido ao oxigênio proteger mais o carbono metínico em orto do que o localizado em posição $\mathrm{para},{ }^{10} \mathrm{o}$ sinal com menor deslocamento químico (em região mais blindada) tornou-se facilmente atribuído ao C-2'. O problema para determinar com relativa segurança a posição ocupada pelo grupo metoxila pode ser resolvido levando-se em consideração o efeito produzido pela metilação nos dois carbonos que ocupam a posição para (C-1' e C-6') em relação à posição que passou pela modificação com a metilação. A comparação dos deslocamentos químicos dos carbonos dos anéis B de 7 e 6 mostrou que o CH-6' de 6 foi pouco afetado, enquanto no C-1' ocorre deslocamento para região
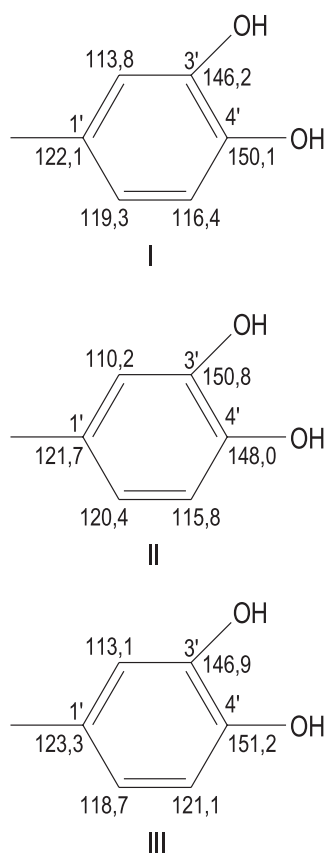

Figura 5. Valores dos deslocamentos químicos para o anel B dos flavonoides com oxigenação em 3'e 4'

mais desblindada de $\Delta \delta_{C}=0,6$ indicando a mudança em C-4'.

Certamente, a acetilação da hidroxila presente no anel B de $\mathbf{1 1}$, fornecendo o derivado 14, foi importante para confirmar inequivocamente os deslocamentos químicos dos carbonos e definir o padrão de substituição da substância $\mathbf{1 1 .}$

O efeito de metilação da hidroxila do C-3' de 11, observado nas substâncias 8, 9, 10, 12 e 13, foi refletido no carbono em posição para, CH-6', que apresentou um deslocamento de $\Delta \delta_{\mathrm{C}}=1,0 \mathrm{ppm}$, e orto, $\mathrm{CH}-2$ ', blindagem de $\Delta \delta_{\mathrm{C}}=3,3 \mathrm{ppm}$.

\section{Caracterização do anel A}

A atribuição dos deslocamentos químicos dos átomos de carbono do anel A apresentou algumas dificuldades quando comparada com a referente ao anel $\mathrm{B}$, em decorrência principalmente da assimetria introduzida pelos dois pontos de ligação do anel A com o anel heterocíclico $\mathrm{C}$.

O sinal de C-10 (não oxigenado) pode ser mais facilmente identificado pela comparação dos espectros de RMN ${ }^{13} \mathrm{C}-\mathrm{HBBD}$ e RMN ${ }^{13} \mathrm{C}$-DEPT (ou APT), permitindo a distinção entre os sinais de carbonos metínicos e quaternários, e pelo seu deslocamento químico comparado com os dos sinais dos carbonos quaternários restantes oxigenados. $\mathrm{O}$ assinalamento dos sinais dos carbonos metínicos $\mathrm{CH}-6 \mathrm{e}$ $\mathrm{CH}-8$ do anel A de flavonóides do tipo floroglucinol é relativammente mais difícil, mas foi monitorado pelo modelo quercetina depois de considerações cuidadosas das correlações em espectro com interação de acoplamento a uma e três ligações $\mathrm{C}-\mathrm{H}^{15}$. Os resultados mostraram que o deslocamento químico do $\mathrm{CH}-8$ aparece com cerca de $\Delta \delta_{\mathrm{C}}=$ 5,0 ppm menor do que o do $\mathrm{CH}-6$. Baseado no efeito que a metilação de HO-3' exerce sobre o carbono vizinho (CH-2'), revelando a diminuição no deslocamento de $\Delta \delta_{\mathrm{C}}=3,3 \mathrm{ppm}$, tornou-se possível o comportamento análogo também para o carbono $\mathrm{CH}-8$ adjacente ao carbono eterificado C-9, revelando-se deslocado para região mais desblindada que o CH-6 adjacente ao carbono C-5 hidroxilado. Sendo assim, a ausência do valor mais baixo $(\approx 93 \mathrm{ppm})$ significa a presença de um substituinte nesta posição, ocorrendo também a correspondente blindagem dos carbonos 7 e 9 por efeito mesomérico. 


\section{Anel A 5,7-dioxigenados}

Os valores dos deslocamentos químicos dos carbonos metínicos CH-6 $\left(\delta_{\mathrm{C}} \sim 98\right)$ e $\mathrm{CH}-8\left(\delta_{\mathrm{C}} \sim 93\right)$ revelam modificação dependente dos grupos substituintes presentes em C-5 e C-7 (Tabela 3 ).

Os espectros de $\mathrm{RMN}^{1} \mathrm{H}$ das substâncias naturais 1-3, 6-9 e 15 revelaram sinal correspondente à hidroxila em ligação de hidrogênio (HO-5) com o C-4 e permitiram reconhecer a presença de função oxigenada adicional no carbono C-7. Os demais flavonoides com o mesmo padrão de oxigenação (5,7-dioxigenados) são os derivados acetilados 4 e $\mathbf{5}$ e $\mathbf{1 0}$ e o metilado 9.

A troca da hidroxila em C-7 do flavonol 7 por uma metoxila, 1-3, 6, 8 e 15 não gerou modificação significativa nos deslocamentos químicos nos carbonos ipso e orto, resultado que pode ser justificado pela variação no uso de solventes na obtenção dos espectros de $\mathrm{RMN}{ }^{13} \mathrm{C}$. O efeito de solvente revelado pela comparação de espectros obtidos em acetona e DMSO permitiu observar desblindagem no carbono da hidroxila fenólica de $\Delta \delta_{\mathrm{C}}=0,6 \mathrm{ppm}$ (carbono ipso) e blindagem de 0,5 no carbono orto, 0,3 no meta e 0,7 para $^{10}$ Os espectros de RMN ${ }^{13} \mathrm{C}$ dos derivados acetilados $\mathbf{4}, \mathbf{5}$ e $\mathbf{1 0}$ revelaram blindagem C-ipso e desblindagem nos carbonos orto e para, como esperado (Tabela 3 ).

\section{Anel A 5,7,8-trioxigenado}

Foram isolados três flavonóis com anel A 5,7,8-trioxigenado, todos com grupo hidroxila em C-5 (11, 12 e 16), e dois foram usados para a preparação dos derivados acetilados 13 e 14, obtendo-se assim produtos com grupo acetoxila em C-5. As outras duas funções oxigenadas poderiam ocupar as posições C-6 e C-7, C-7 e C-8 ou C-6 e C-8 deste anel. A última alternativa foi descartada através de argumentação biogenética e pelo deslocamento químico apresentado pelo C-10, que é praticamente o mesmo dos flavonóis com anel A dioxigenado com oxigenação em $\mathrm{C}-7$, que protege $\mathrm{C}-10$ por efeito mesomérico. A caracterização do anel A dos flavonóis com padrão de oxigenação 5,7,8-trioxigenados ou 5,6,7-trioxigenados não pode ser definida com a necessária garantia pelo valor de deslocamento químico do carbono metínico, já que foi encontrado um valor médio $\left(\delta_{\mathrm{C}} 95\right)$ para os deslocamentos químicos de $\mathrm{CH}-6$ e $\mathrm{CH}-8$, observandose uma diferença de aproximadamente $\Delta \delta_{C}=3$ ppm que pode ser tanto para o carbono $\mathrm{CH}-6$ como para o $\mathrm{CH}-8$ (como modelo podese citar a substância 8). Entretanto, os espectros de $\mathrm{RMN}{ }^{13} \mathrm{C}$ dos derivados acetilados 13 e 14, obtidos através de reação de acetilação dos flavonóis 11 e 12 (conversão da hidroxila em C-5 em acetoxila) revelaram, como esperado, os sinais dos carbonos em posição orto (CH-6 e C-10) e para (C-8) com maiores valores de deslocamento químico $\left(\delta_{\mathrm{CH}-6} 104,00 ; \delta_{\mathrm{CH}-10} 111,70\right)$, que demonstram, claramente, a menor densidade eletrônica nestes carbonos (atenuação do efeito mesomérico doador de elétrons do átomo de oxigênio ligado ao C-5). Com base nestes valores de deslocamentos químicos foi possível assegurar inequivocamente o carbono metínico como sendo $\mathrm{CH}-6$ e o quaternário C-10. Os carbonos localizados em posição para (C-8) também foram afetados, revelando uma diferença de deslocamento para região mais desblindada de $\Delta \delta_{\mathrm{C}}=6,0 \mathrm{ppm}$.

\section{Aplicação de técnicas 1D (NOEDIFF e DEPT) e experiências bidimensionais (2D)}

A atribuição inequívoca dos $\delta_{\mathrm{H}}$ e $\delta_{\mathrm{C}}$ das substâncias naturais $\mathbf{1 , 2}$, 6-8, 11, 12, 15 e 16 apoiou-se também nas técnicas de 2D de RMN e nas modificações observadas nos dados espectrais dos derivados 3-5, 9-10, 13-14. Estes derivados também forneceram informações adicionais importantes para elucidação estrutural.

Os espectros de RMN ${ }^{13} \mathrm{C}$-DEPT $135^{\circ}$ das substâncias naturais 1 e 2, apresentaram 5 (1) e 6 (2) sinais de carbonos hidrogenados, sendo quatro atribuídos a carbonos metínicos (1 e 2), uma metoxila (1) e duas metoxilas (2). Os espectros de HMQC permitiram estabelecer as correlações diretas $\left({ }^{1} \mathrm{~J}_{\mathrm{CH}}\right)$ : $\mathrm{CH}-6\left[\delta_{\mathrm{H}} / \delta_{\mathrm{C}} 6,35 / 97,77\right.$ (2) e 6,32/97,47(1)], $\mathrm{CH}-8$ $\left[\delta_{\mathrm{H}} / \delta_{\mathrm{C}} 6,74 / 92,37(\mathbf{2})\right.$ e $\left.6,71 / 92,02(\mathbf{1})\right] ; 2 \mathrm{CH}-3,, 5\left[\delta_{\mathrm{H}} / \delta_{\mathrm{C}} 6,92 / 115,68(\mathbf{2})\right.$ e 6,32/115,47(1)]; 2CH-2',6' [ $\delta_{\mathrm{H}} / \delta_{\mathrm{C}} 7,96 / 130,24(\mathbf{2})$ e 8,06/129,61(1)]. As posições das metoxilas foram estabelecidas pelos espectros $\mathrm{HMBC}$ : $\mathrm{MeO}-7 / \mathrm{C}-7,{ }^{3} \mathrm{~J}_{\mathrm{CH}}\left[\delta_{\mathrm{H}} / \delta_{\mathrm{C}} 3,84 / 164,91\right.$ (1) e 3,84/165,13 (2)]; H-6/C-7, ${ }^{2} \mathrm{~J}_{\mathrm{CH}}\left[\delta_{\mathrm{H}} / \delta_{\mathrm{C}} 6,32 / 164,91(\mathbf{1})\right.$ e $\left.6,35 / 165,13(\mathbf{2})\right]$ e H-8/C-7, ${ }^{2} \mathrm{~J}_{\mathrm{CH}}\left[\delta_{\mathrm{H}} / \delta_{\mathrm{C}}\right.$ 6,71/164,91 (1) e 6,74/165,13 (2)] e MeO-3/C-3, ${ }^{3} \mathrm{~J}_{\mathrm{CH}}[3,78 / 137,86$ (2)]. A acetilação deste flavonol (1) forneceu o derivado 5, que foi usado também para obtenção do espectro de NOESY e observar mais uma vez a localização da metoxila em C-7 pelas interações espaciais com os hidrogênios H-6 $\left(\delta_{H} 6,65\right)$ e H-8 $\left(\delta_{H} 6,84\right)$.

A interpretação dos resultados obtidos pelas experiências $2 \mathrm{D}$ de RMN dos derivados seguiu o mesmo procedimento e forneceu informações adicionais úteis.

Os espectros de $\mathrm{RMN}{ }^{1} \mathrm{H}\left(1 \mathrm{D}\right.$ e $\left.2 \mathrm{D}{ }^{1} \mathrm{H}-{ }^{1} \mathrm{H}-\mathrm{COSY}\right)$ dos flavonóis 6 , 7 e 8 revelaram sinais de átomos de hidrogênio atribuídos aos sistemas $\mathrm{AB}$ do anel $\mathrm{A}$ e $\mathrm{ABC}$ do anel $\mathrm{B}$. Estes sistemas foram confirmados pelo espectro $2 \mathrm{D}{ }^{1} \mathrm{H}-{ }^{1} \mathrm{H}-\mathrm{COSY}$ de 7 através de picos transversais correspondentes às interações spin-spin do H-6 $\left(\delta_{\mathrm{H}} 6,25\right)$ com H-8 $\left(\delta_{\mathrm{H}} 6,48\right)$ e do H-6' $\left(\delta_{\mathrm{H}} 7,57\right)$ com H-2' e H-5'. Os espectros de RMN ${ }^{1} \mathrm{H}$ e $\mathrm{RMN}{ }^{13} \mathrm{C}\left(\left\{{ }^{1} \mathrm{H}\right\}\right.$ e DEPT $)$ indicaram a presença de uma $\mathrm{MeO}$ em 7, quatro em $\mathbf{8}$ e $\mathbf{1 0}$ e cinco em 9. Os espectros HMQC de $\mathbf{7}$ e $\mathbf{8}$ foram usados para estabelecer as correlações dos carbonos metínicos e metoxilicos (uma em 7 e quatro em 8). $\mathrm{O}$ valor do deslocamento químico do carbono metoxílico $\left(\delta_{\mathrm{C}} 58,84\right)$ e o espectro HMBC através da correlação do MeO-3 $\left(\delta_{\mathrm{H}} 3,86\right)$ com C-3 $\left(\delta_{\mathrm{C}} 137,94,{ }^{3} \mathrm{~J}_{\mathrm{CH}}\right)$ indicaram indubitavelmente a presença da metoxila em C-3 do flavonoide 7. O espectro de $\mathrm{RMN}{ }^{1} \mathrm{H}$ do flavonoide $\mathbf{8}$ mostrou, além dos sinais das metoxilas (com integração para $4 \mathrm{MeO}$ ) e dos sistemas $\mathrm{AB}$ e $\mathrm{ABC}$, sinal de HO-5 (hidroxila em ligação de hidrogênio com a carbonila no C-4), permitindo a localização das 4 metoxilas nos carbonos C-3, C-7, C-3' e C-4', mesmo sem conhecer até este ponto os valores dos deslocamentos químicos dos átomos de hidrogênio de cada uma. Os picos transversais correspondentes às interações heteronucleares $\left({ }^{3} J_{\mathrm{CH}}\right)$ de C-3 $\left(\delta_{\mathrm{C}} 139,40\right)$ com MeO-3 $\left(\delta_{\mathrm{H}} 3,87\right), \mathrm{C}-7\left(\delta_{\mathrm{C}} 165,86\right) \mathrm{com}$ MeO-7 $\left(\delta_{\mathrm{H}} 3,89\right), \mathrm{C}-3$ ' $\left(\delta_{\mathrm{C}} 149,18\right)$ com MeO-3' $\left(\delta_{\mathrm{H}} 3,97\right)$ e C-4' $\left(\delta_{\mathrm{C}}\right.$ $151,79)$ com MeO-4' $\left(\delta_{\mathrm{H}} 3,98\right)$ observados no espectro HMBC permitiram definir o valor do deslocamento químico para cada metoxila e de cada carbono não hidrogenado. O espectro de NOESY mostrou interações espaciais das metoxilas MeO-7 $\left(\delta_{\mathrm{H}} 3,89\right)$ com H-6 $\left(\delta_{\mathrm{H}} 6,37\right)$ e H-8 $\left(\delta_{\mathrm{H}} 6,46\right), \mathrm{MeO}-3$ ' $\left(\delta_{\mathrm{H}} 3,87\right)$ com H-2' $\left(\delta_{\mathrm{H}} 7,70\right)$ e MeO-4' $\left(\delta_{\mathrm{H}}\right.$ $3,98)$ com H-5' $\left(\delta_{\mathrm{H}} 7,00\right)$, confirmando, portanto, as atribuições dos $\delta_{\mathrm{H}}$ dos grupos metoxílicos.

Os espectros de $\mathrm{RMN}{ }^{1} \mathrm{H}$ do flavonol 6 revelaram sinais para os sistemas $\mathrm{AB}$ e $\mathrm{ABC}$ e para duas metoxilas em $\delta_{\mathrm{H}} 3,92$ e 3,93. As interações dipolares de $\mathrm{MeO}-4$ ' $\left(\delta_{\mathrm{H}} 3,93\right)$ com H-5' $\left(\delta_{\mathrm{H}} 7,11\right)$ e $\mathrm{MeO}$ $7\left(\delta_{\mathrm{H}} 3,92\right)$ com H-6 $\left(\delta_{\mathrm{H}} 6,31\right)$ e H-8 $\left(\delta_{\mathrm{H}} 6,68\right)$ foram observadas no espectro 2D NOESY, confirmando a caracterização estrutural de $\mathbf{6}$ como 3,3',5-triidroxi-4',7-dimetoxiflavona.

O flavonol permetilado 9 mostrou sinais de interação espacial no espectro de NOESY das metoxilas MeO-7 $\left(\delta_{\mathrm{H}} 3,93\right)$ com H-8 $\left(\delta_{\mathrm{H}}\right.$ $6,57), \mathrm{MeO}-5\left(\delta_{\mathrm{H}} 3,96\right)$ com H-6 $\left(\delta_{\mathrm{H}} 6,39\right), \mathrm{MeO}-3$ ' $\left(\delta_{\mathrm{H}} 3,98\right) \mathrm{com}$ H-2' $\left(\delta_{\mathrm{H}} 7,74\right)$ e MeO-4' $\left(\delta_{\mathrm{H}}\right)$ com H-5' $\left(\delta_{\mathrm{H}} 7,02\right)$.

A principal diferença observada na comparação dos espectros de $\mathrm{RMN}{ }^{1} \mathrm{H}$ dos flavonóis $\mathbf{1 1}$ e $\mathbf{1 2}$ foi atribuída à presença de uma metoxila adicional em 12. Os anéis A de $\mathbf{1 1}$ e $\mathbf{1 2}$ contêm somente um átomo de hidrogênio isolado. Os sinais simples correspondentes a estes átomos de hidrogênio aparecem em $\delta_{H} 6,41(\mathbf{1 1})$ e $\delta_{H} 6,43$ (12) nos espectros de $\mathrm{RMN}{ }^{1} \mathrm{H}$ e apresentaram correlações com os carbonos metínicos em $\delta_{\mathrm{C}} 95,83$ e 95,60, respectivamente, nos espectros de HMQC. O espectro HMBC de $\mathbf{1 1}$ mostrou correlação do H-6 $\left(\delta_{\mathrm{H}} 6,41\right)$ através de duas ligações $\left({ }^{2} J_{\mathrm{CH}}\right)$ com os carbonos 
C-5 $\left(\delta_{\mathrm{C}} 157,49\right)$ e C-7 $\left(\delta_{\mathrm{C}} 158,56\right)$ e de três ligações $\left({ }^{3} J_{\mathrm{CH}}\right)$ com C-8 $\left(\delta_{\mathrm{C}} 129,07\right)$ e $\mathrm{C}-10\left(\delta_{\mathrm{C}} 105,53\right)$. Estes dados permitiram assegurar a localização da metoxila adicional do anel A no carbono C-8.

O NOE $(8 \%)$ observado no sinal de $\mathrm{H}^{-} 5^{\prime}\left(\delta_{\mathrm{H}} 7,10\right)$ do derivado acetilado 14, revelado pela irradiação na frequência de absorção dos hidrogênios metoxílicos representados pelo simpleto em $\delta_{\mathrm{H}} 3,96$ (MeO-4') no espectro de $\mathrm{RMN}{ }^{1} \mathrm{H}$, serviu para confirmar a presença da metoxila em C-4'. Irradiação nas frequências de absorção dos átomos de hidrogênio das duas metoxilas em $\delta_{\mathrm{H}} 3,87(\mathrm{MeO}-3)$ e 3,94 (MeO-7) mostraram NOE de 1 e $2 \%$ nos sinais do H-2' $\left(\delta_{\mathrm{H}} 7,78\right)$ e H-6' $\left(\delta_{\mathrm{H}} 7,76\right)$ e de $5 \%$ em H-6 $\left(\delta_{\mathrm{H}} 6,41\right)$, respectivamente.

$\mathrm{O}$ espectro de NOESY do flavonol 12 mostrou interação das metoxilas em MeO-7 ( $\delta_{\mathrm{H}} 3,96, \mathrm{MeO}-3$ ' $(\delta 3.98)$ e MeO-4' $\left(\delta_{\mathrm{H}} 3,99\right)$ com os átomos de hidrogênio H-6 $\left(\delta_{\mathrm{H}} 6,43\right), \mathrm{H}-2$ ' $\left(\delta_{\mathrm{H}} 7,82\right)$ e H-5' $\left(\delta_{\mathrm{H}} 7,03\right)$, respectivamente. Estas informações serviram mais uma vez para confirmação das posições destas metoxilas.

Todos os flavonois naturais isolados de Solanum paludosum e $S$. jabrense encontram-se descritos na literatura, porém poucos ou nenhum dados espectroscópicos existiam para as substâncias. Os dados de RMN ${ }^{1} \mathrm{H}$ ou ${ }^{13} \mathrm{C}$ para os flavonoides $1,9,162,{ }^{17} \mathbf{6},{ }^{9} 7,{ }^{9} 8,{ }^{18} \mathbf{1 2},{ }^{19} \mathbf{1 5},{ }^{10} \mathrm{e} \mathbf{1 6}{ }^{20}$ foram comparados com os da literatura. Os flavonoides 1, 6, 11, 12, 15 e 16 foram registrados pela primeira vez no gênero Solanum.

\section{AGRADECIMENTOS}

Ao CNPq, CAPES e FAPERJ pelas bolsas e auxílios financeiros concedidos, ao Setor de Química de Produtos Naturais (SQPN), Laboratório de Ciências Químicas (LCQUI), Centro de Ciência e Tecnologia (CCT) da Universidade Estadual do Norte Fluminense Darcy Ribeiro (UENF) - Campus Leonel Brizola/Campos dos Goytacazes - RJ e ao Centro Nordestino de Aplicação e Uso de Ressonância Magnética Nuclear (CENAUREMN)-Programa de Pós-graduação em Química Orgânica (PPGQO), Departamento de Química Orgânica e Inorgânica, Centro de Ciências da Universidade Federal do Ceará, Fortaleza-CE, pelos espectros 1D e 2D de Ressonância Magnética Nuclear (RMN ${ }^{1} \mathrm{H}$ e RMN $\left.{ }^{13} \mathrm{C}\right)$.

\section{REFERÊNCIAS}

1. Silva, T. M. S.; Braz-Filho, R.; Carvalho, M. G.; Agra, M. F.; Biochem. Syst. Ecol. 2002, 30, 479.
2. Silva, T. M. S.; Nascimento, R. J. B.; Camara, C. A.; Agra, M. F.; BrazFilho, R.; Carvalho, M. G.; Biochem. Syst. Ecol. 2004, 32, 513.

3. Silva, T. M. S.; Tese de Doutorado, Universidade Federal Rural do Rio de Janeiro, Brasil, 2002.

4. Camara, C. A.; Pinto, A. C.; Vargas, M. D.; Tetrahedron 2002, 58, 6135.

5. Mabry, T. J.; Markam, K. R.; Thomas, M. B.; The systematic identification of flavonoids, Springer: New York, 1970.

6. Fossen, T.; Andersen O. M. Em Flavonoids Chemistry, Biochemistry and Applications: Spectroscopic Techniques Applied to Flavonoids; Anderson, O. M.; Markham, K. R., eds.; Taylor \& Francis: New York, 2006, p. 37-142.

7. Goudard, M.; Favre-Bonvin, J.; Lebreton, P.; Chopin, J.; Phytochemistry 1979, 18, 186.

8. Markham, K. R. Em Methods in plant biochemistry: Flavones, flavonols and their glycosides; Harborne, J. B.; Mabry, T. J., eds.; Academic Press: New York, 1989, p. 197-235.

9. Agrawal, P. K. E.; Carbon-13 NMR of Flavonoids, Studies in Organic Chemistry-39: Methods in Signal Assignement, Elsevier: Amsterdam, 1989.

10. Markham, K. R.; Chari, M.; Mabry, T. J. Em The Flavonoids: Advances in Research: Carbon-13 NMR Spectroscopy of Flavonoids; Harborne, J. B.; Mabry, T. J., eds.; Chapman \& Hall: London, 1982, p. 19-134.

11. Mabry, T. J.; Markham, K. R. Em The Flavonoids; Harborne, J. B.; Mabry, T. J., eds.; Academic Press: New York, 1975.

12. Kingsbury, C. A.; Looker, J. H.; J. Org. Chem. 1975, 40, 1120.

13. Pelter, A.; Ward, R. S.; Gray, T. I.; J. Chem. Soc., Perkin Trans. 1976, 1, 2475.

14. Bohm, B. A.; Introduction to Flavonoids, Harwood Reading: Amsterdam, 1999, p. 212.

15. Lallemand, J. Y.; Duteil, M.; Org. Magn. Reson. 1977, 9, 179.

16. Barberá, O.; Sanz, J. F.; Sánchez-Parareda, J.; Marco, J. A.; Phytochemistry 1986, 25, 2361.

17. Parsons, I. C.; Gray, A. I.; Waterman, P. G.; Hartley, T. G.; J. Nat. Prod. 1993, 56, 46 .

18. Li, S.; Lo, C-Y.; Ho, C-T.; J. Agric. Food Chem. 2006, 54, 4176.

19. Ferracin, R. J.; Silva, M. F. G. F.; Fernandes, J. B.; Vieira, P. C.; Phytochemistry 1998, 47, 393.

20. Horie, T.; Ohtsuru, Y.; Shibata, K.; Yamashita, K.; Tsukayama, M.; Kawamura, Y.; Phytochemistry 1998, 47, 865. 